Planetary Systems in the Universe - Observation, Formation and Evolution

Proceedings IAU Symposium No. 202, (c)2004 IAU

Alan Penny, Pawel Artymowicz, Anne-Marie Lagrange, \& Sara Russell, eds.

\title{
Can D-D fusion contribute to Jupiter's excess heat?
}

\author{
Rachid Ouyed \\ NORDITA, Blegdamsvej 17, DK-2100 Copenhagen, Denmark
}

\begin{abstract}
We explore the highly speculative idea of Deuterium - Deuterium (D-D) fusion inside Jupiter as an internal heating source. We suggest that D could have been brought deep inside the planet by planetesimals (during the process of planet formation) and deposited through planetesimal/ices vaporization. Here, the general aspects of the model are presented.
\end{abstract}

\section{Introduction}

The most common explanation for Jupiter's excess heat $\left(P_{\text {obs. }} \sim 3 \times 10^{24} \mathrm{erg}\right.$ $\mathrm{s}^{-1}$ ) is through the release of gravitational potential energy associated with the planet's contraction. In such a model, the planet is convective and of homogeneous composition and thus exhibit adiabatic thermal gradients to transport efficiently its internal energy (for e.g., Hubbard 1984, Podolak et al. 1993). However, the first observations of global free oscillations of Jupiter, suggest that there is a discrepancy between the calculated sound velocity and the Jovian sound profiles (Mosser et al., 1993; Duffy et al., 1994). The difficulty faced by theoretical models in trying to reproduce the observed seismological properties of Jupiter (like the sound velocity estimate that result from the current interpretation of the Jovian global oscillations ${ }^{1}$ ) suggest either a revision of the hypothesis of a homogeneous distribution of elements within each part of the envelope, or a bias in the observations (Gudkova et al. 1995). In other words, a nonstandard view of Jupiter's interior might be the only way to reconciliate theoretical and observed values (Mosser et al., 2000). One such model was presented in Ouyed et al. (1998) and is further discussed here.

\section{D-D fusion inside Jupiter?}

In Ouyed et al. (1998), we investigated the possibility of D-D fusion inside Jupiter as an internal heat source and its consequences on the internal structure of the planet. Such interest resulted from the recent extrapolations of D-D, $\mathrm{D}-\mathrm{T}, \mathrm{D}-{ }^{3} \mathrm{He}$ and $\mathrm{p}-\mathrm{D}$ fusion to $\mathrm{eV}$ temperatures which indicate that $\mathrm{D}-\mathrm{D}$ fusion largely dominates the other reactions (see Figure 1 in Ouyed et al., 1998). It is straightforward to show that in a homogeneous composition (as it is assumed in

\footnotetext{
${ }^{1}$ The frequency of the measured oscillations is $\sim 20 \%$ less than expected for current models. If these oscillations are, in fact, seismic oscillations, then sound velocities $\left(c_{S}^{2}=(\partial P / \partial \rho)_{S}\right.$ where $S$ is the entropy) $\sim 20 \%$ less than currently calculated over the entire planet must be explained (Nellis et al. 1995).
} 
the conventional models of Jupiter), D-D fusion is negligible when compared to $\mathrm{p}$ - $\mathrm{D}$ fusion because of the $\mathrm{D} / \mathrm{H}=10^{-5}$ dilution effect. However, if $\mathrm{D}$ manages to sediment into a layer with a dilution factor of $10^{-3}$, then D-D fusion would be at least as important as the p-D fusion (see Ouyed et al., 1998 for details). If an even less diluted layer of $\mathrm{D}$ forms, than D-D becomes a plausible energy source that might contribute to the excess heat in Jupiter.

For D-D fusion to contribute significantly to the excess heat, we found that simplified D distributions imply i) central temperatures $\left(T_{\mathrm{c}}\right)$ of the order of $16-18 \mathrm{eV}$ (discussed in the conclusion) and ii) $5 \%-15 \%$ of the total $\mathrm{D}$ of the planet to have stratified into the planet's interior (discussed in $\S 3$ ). The total energy that is released in D-D fusion can be estimated as $E_{D} \simeq 0.005 M_{D} c^{2} \times$ $\gamma_{D} \simeq 10^{43} \mathrm{erg}\left(M_{D}\right.$ is the total $\mathrm{D}$ in the planet of which a percentage $\gamma_{D}$ had stratified into a layer). If no more than $15 \%\left(\gamma_{D}=0.15\right)$ of the total $\mathrm{D}$ concentrates in the D layer, it would be exhausted in $E_{D} / P_{e x c} \simeq 10^{18} s \simeq 10^{11}$ years, if consumed at the present rate of excess heat observed. The resulting cycle, we refer to as the D-D cycle, is discussed in length in Ouyed et al. (1998).

\section{Planetesimal vaporization and $D$ sedimentation}

We estimated that $5 \%-15 \%$ of the total $\mathrm{D}$ of the planet is required to stratify into the interior of the planet, or equivalently, the corresponding amount of D contained in the $\mathrm{D}$ layer $\left(M_{\mathrm{D}, \text { Layer }}\right)$ is approximately,

$$
10^{-4} M_{\oplus} \leq M_{D, \text { Layer }} \leq 3.0 \times 10^{-4} M_{\oplus}
$$

assuming $\mathrm{D} / \mathrm{H}=2.0 \times 10^{-5}$ over the whole planet. But, how much condensed material (especially ices) did Jupiter accrete during its formation?

If we consider that about $10 M_{\oplus}$ in ices fell onto Jupiter's core, and if we take the $\mathrm{D}$ content of these icy planetesimals to be on average $\mathrm{D} / \mathrm{H} \simeq 4.0 \times 10^{-4}$ (Owen et al. 1986; Lutz et al. 1990), than the amount of D brought in deep in the envelope is roughly, $M_{D \text {,ices }} \sim 4.0 \times 10^{-3} M_{\oplus}$. Hence, to account for the amount of $\mathrm{D}$ required in a layer, as given by eq. (1), we require vaporization efficiency $\zeta$ in the range:

$$
5 \% \leq \zeta \leq 10 \%,
$$

during Jupiter formation. Unfortunately, however, there are no definite numbers as for the vaporization efficiency and the distribution of the vaporized elements remains to be calculated; the whole issue is complicated by the core-envelope structure and by the variation of the opacity with temperature and density. A more complete study needs to incorporate the effect of dissolved planetesimals on the properties of the envelope itself, such as the convective instability and the effects of occasional massive impacts (e.g. due to very massive planetesimals) before any final conclusions can be reached. Finally, let us mention that the superficial depletion of D (even as high as $30 \%$ of the total D of the planet) may be observationally hard to detect. 


\section{Conclusion}

For D-D fusion to be at all significant, central temperatures of the order of $16-18 \mathrm{eV}$ are required! Even if such high temperatures are possible (unlikely in standard models of Jupiter formation), we still need to invoke a totally different equation of state, and thus composition, for the interior of Jupiter (Chabrier et al., 1992). With an adequate equation of state, one must also reproduce the observed condition of Jupiter, i.e. $T_{1}$ bar $=165 \mathrm{~K}$, for the correct mass and radius; this is work in progress. For now, one should keep in mind the fact that measurements and extrapolation of light elements fusion cross-section to $\mathrm{eV}$ temperatures are constantly updated ${ }^{2}$; it might turn out that one would not need to go to such high temperatures for the excess heat to be explained by D-D fusion. At a more fundamental level, a detailed treatment of element sedimentation (in particular $\mathrm{D}$ ) through planetesimal vaporization will be the ultimate test in whether or not our model is at all possible.

\section{References}

Bosch, H. S., \& Hale, G. M. 1992, Nuclear Fusion, 32, 611

Chabrier, G., Saumon, D., Hubbard, W. B. \& Lunine, J. I. 1992, ApJ, 391, 817

Duffy, T. S., Vos, W. L., Zha, C.-S., Hemley, R. J. \& Mao, H.-K. 1994, Science, 263, 1590

Gudkova, T. V., Mosser, B., Provost, J., Chabrier, G., Gautier, D. \& Guillot, T. 1995, A\&A, 303, 594

Hubbard, W. B., 1984, Planetary Interiors. New York: Van Nostrand Reinhold.

Lutz, B. L., Owen, T. \& de Bergh, C. 1990, Icarus, 86, 329

Mosser, B., Mékarnia, D., Maillard, J. P., Gay, J., Gauthier, D. \& Delache, Ph., 1993, A\&A, 267, 604

Mosser, B., Maillard, J. P. \& Mékarnia, D. 2000, Icarus, 144, 104

Nellis, W. J., Ross, M. \& Holmes, N. C. 1995, Science, 269, 1249

Ouyed, R., Fundamenski, W., Cripps, G. \& Sutherland, P. G. 1998, ApJ, 501, 367

Owen, T., Lutz, B. L. \& de Bergh, C. 1986, Nature, 320, 244

Podolak, M., Hubbard, W. B. \& Pollack, J. B. 1993, in Protostars and planets III, Levy, E. H. and Lunine, J. I., eds., University of Arizona Press, Tucson, 1109

\footnotetext{
${ }^{2}$ Empirical measurements of fusion reaction rates are only possible at energies approaching the Coulomb barrier, i.e in the tens of $\mathrm{keV}$ range. However, these results can be extrapolated to the lower $\mathrm{eV}$ temperatures with the use of quantum mechanical scattering theory. The latest study employs R-matrix scattering theory based on compound nucleus formation and examines the data from all reaction channels (Bosch \& Hale 1992). For example, the ${ }^{4} \mathrm{He}$ compound nucleus produced during D-D fusion can also be obtained via $\mathrm{p}-{ }^{3} \mathrm{He}$ and $n-{ }^{3} \mathrm{He}$ channels. As such, the data set is greatly extended and the fusion cross-sections constantly updated.
} 